\title{
Enhancement of Color Image Retrieval using Adaptive Texture Descriptor
}

\author{
Disha Sugha \\ Department of Electronics \& \\ Communication \\ Sri Sai University, Palampur \\ India
}

\author{
Honeyily \\ Department of Electronics \& \\ Communication \\ Sri Sai University, Palampur \\ India
}

\author{
Vinay Thakur \\ Department of Electronics \& \\ Communication \\ Sri Sai University, Palampur \\ India
}

\begin{abstract}
Objective of finding digital images from large database various methods of computer vision techniques of image retrieval are there, out of which Content Based Image Retrieval (CBIR) is widely used. Modern image retrieval systems use content-based image retrieval, as there is increased demand of images in digital format. Nowadays, the latest technique used for image retrieval is content based image retrieval. In digital image processing CBIR is rapidly growing field. In content-based image retrieval method, retrieval of image requires various visual features of image, like- color, shape, texture, etc; so that desired image could be retrieved as per the requirement of user. On the other hand, if we talk about retrieval of image manually from huge set of databases, is time-consuming, laborious and expensive as compared to content-based image retrieval. Therefore, to reduce time for image retrieval we prefer CBIR. In this paper, technique for content-based image retrieval includes texture of data image which is used to calculate energy, contrast and time elapsed of an image using adaptive texture descriptor method. Hence, to increase the enhancement of image, number of features increased for comparison with previous work and value of parameters are also calculated for comparative analysis and on comparing features with stored database time elapsed is also calculated.
\end{abstract}

\section{General Terms}

Feature Extraction, texture descriptor, color histogram, Elapsed time, Energy, Contrast.

\section{Keywords}

Graphical user interface (GUI), Content based image retrieval (CBIR), RGB to Gray.

\section{INTRODUCTION}

In image processing field content-based image retrieval begin to become an important and widely used topic for researches as there is swift increase of digital format in database which consists of images and videos. Content based image retrieval is vital in order to avoid traditional methods (such as image searching based on texts) of image retrieval which are more time-consuming and laborious, and with the help of contentbased image retrieval method users could enhance the quality of image or information as per the requirement. In text-based image retrieval, images are retrieved using texts which are also known as metadata of image. As it is manual process of retrieving images, it is more time-consuming and it cannot represent visual contents of image like color, texture and shape etc. [2]. There are chances of inaccuracy and fault in retrieving images as it works manually to interpret every single image. Many complications with text-based access to images results in content-based image retrieval method of image retrieval to overcome. The main focus of CBIR is to abandon the use of text base image retrieval method. CBIR makes the user to handle the data more easily, thereby, making the data user friendly for the user. The quick retrieval of images from large set of databases is achieved with the help of content-based image retrieval. In the process of retrieving the image from large database, system needs to search the images which are to be rationally same as a query images with the help of CBIR [4]. Content based image retrieval methods are used to retrieve an effective and an appropriate image from database. Commonly used contentbased image retrieval methods work in a way by extracting low level features such as color, texture, shapes, range to do analysis by comparing the data image with query image. CBIR is also used for calculation of feature vector of characteristics of database image. This feature vector calculations requires query image and feature vector is calculated using CBIR and after that comparing it with the images in database.

\subsection{Parameters of Image}

In this paper we have done work on various parameters which energy, contrast, time elapsed to retrieve image, histogram and local range and these parameters are discussed below:

\subsubsection{Energy/ Entropy}

The difference between the contiguous regions of image gives the energy of image. The greater the pixel value or group of pixels of image, the higher the value of entropy of an image. Mathematically, we can say that it is sum of square of elements in gray-level co-occurrence matrix (GCLM).

\subsubsection{Contrast}

For the enhancement of quality of image contrast is considered to be important parameter. The difference between luminance bounced back from two adjoining surfaces gives the value of contrast [8]. Contrast is defined as the difference in color and brightness of image with the same field of view or background.

\subsubsection{Time Elapsed}

Time elapsed is defined as the interval from the time taken to start the task till the task is completed. If we talk about time elapsed in image retrieval, it is the time required to retrieve the image from large database.

\subsubsection{Histogram}

The intensity of pixels of image represented in graphical format is known as histogram. It generally shows the maximum and minimum value of pixel of an image [4].

\subsubsection{Local Range}

In adjoining surface the pixel range value is small whereas in rough texture area the pixel range value is larger. 


\section{PROPOSED METHODOLOGY}

Color and texture of an image from database has been primarily used to retrieve images from the retrieval system. In this paper adaptive texture descriptor method is used to calculate three parameters of image which are energy, contrast and time elapsed to retrieve image. The steps of methodology are as follows:

[1] To close all pre-open window.

[2] Load database in MATLAB workspace.

[3] To initialize input variation range, it should be negative or zero.

[4] Convert image from RGB to Gray.

[5] Find entropy, contrast and time elapsed for image.

[6] Combine the image features.

[7] Load test image.

[8] Apply procedure 3-6 to find combine feature of test image.

[9] To calculate elapsed time when features comparing with stored database.

[10] Display the result on GUI.

\subsection{Objectives}

[1] To extract features of image from the database.

[2] To calculate numerical value of parameters for comparative analysis such as contrast, energy, and time elapsed.

[3] To obtain the results in GUI as to make the output user friendly.

\section{ANALYSIS OF RESULT}

The developed algorithm is tested for various test images. Database consists of different color and texture-based image. The results obtained are shown in table.

\subsection{Image Retrieval is Proposed with Adaptive Texture Descriptor Method}

Value of texture of image from large database of images has been calculated. In this paper value of energy, contrast and elapsed time to retrieve the image is calculated. During the process of image retrieval, we require a query image and the features of this query image are being extracted and after that these features are matched with the features of image in database [5]. Firstly, the database is loaded in MATLAB and after that we resize the image from database to obtain the same size of images. Further we convert images from RGB to Gray format. Then we normalize the gray image for fixed mean and after this we find the histogram, local range, energy and time elapsed of each image [6].The closest values are displayed on GUI as result.
Table 1. Parameters of proposed algorithm

\begin{tabular}{|c|c|c|c|}
\hline Test Images & $\begin{array}{c}\text { Evaluation } \\
\text { metrics }\end{array}$ & $\begin{array}{c}\text { Previous } \\
\text { Approach }\end{array}$ & $\begin{array}{l}\text { Proposed } \\
\text { Approach }\end{array}$ \\
\hline \multirow{3}{*}{ Image 1} & Energy & $5.25616 \mathrm{e}-005$ & 7.40186e-005 \\
\hline & Contrast & 1828.91 & 2342.01 \\
\hline & $\begin{array}{c}\text { Elapsed } \\
\text { Time }\end{array}$ & 30.6328 & 26.9958 \\
\hline \multirow{3}{*}{ Image 2} & Energy & $5.12588 \mathrm{e}-005$ & $8.84614 \mathrm{e}$ \\
\hline & Contrast & 20.83 .39 & 1900.36 \\
\hline & $\begin{array}{l}\text { Elapsed } \\
\text { Time }\end{array}$ & 28.6623 & 25.4171 \\
\hline \multirow{3}{*}{ Image 3} & Energy & $6.56541 \mathrm{e}$ & $9.94021 \mathrm{e}-005$ \\
\hline & Contrast & 1960.86 & 2283.6 \\
\hline & $\begin{array}{l}\text { Elapsed } \\
\text { Time }\end{array}$ & 24.6873 & 23.513 \\
\hline \multirow{3}{*}{ Image 4} & Energy & 0.000101475 & 0.000122932 \\
\hline & Contrast & 1463.19 & 1976.29 \\
\hline & $\begin{array}{c}\text { Elapsed } \\
\text { Time }\end{array}$ & 26.0229 & 23.8763 \\
\hline \multirow{3}{*}{ Image 5} & Energy & 0.000101475 & 0.000122932 \\
\hline & Contrast & 1463.19 & 1976.29 \\
\hline & $\begin{array}{l}\text { Elapsed } \\
\text { Time }\end{array}$ & 28.34 & 21.2804 \\
\hline
\end{tabular}

\section{STIMULATED RESULTS}

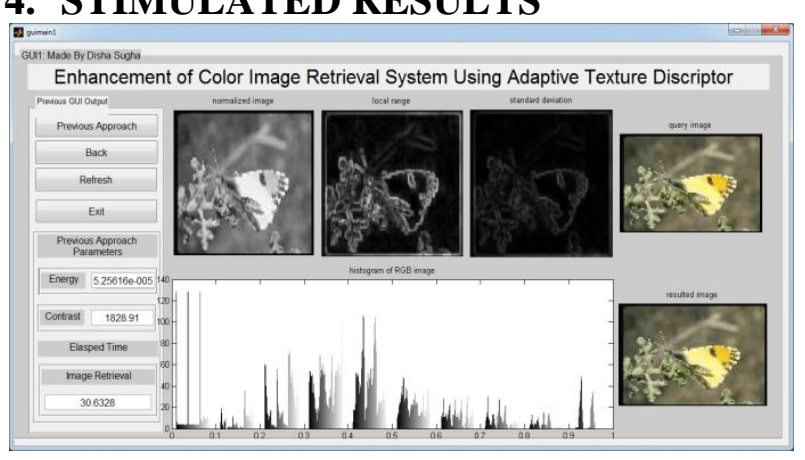

Fig1. GUI1 previous approach

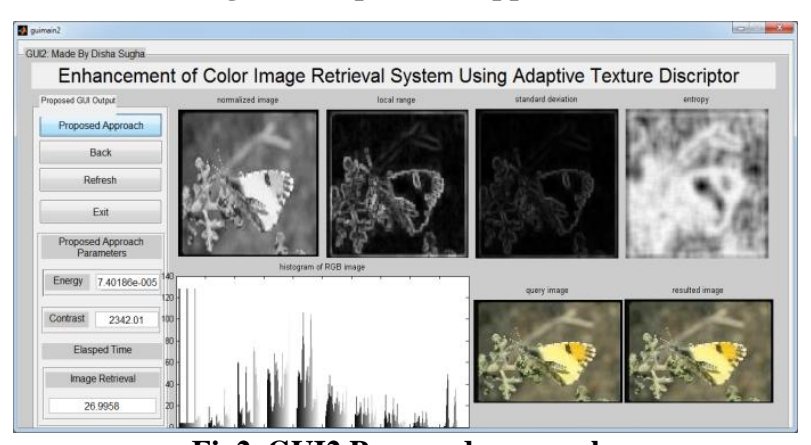

Fig2. GUI2 Proposed approach 


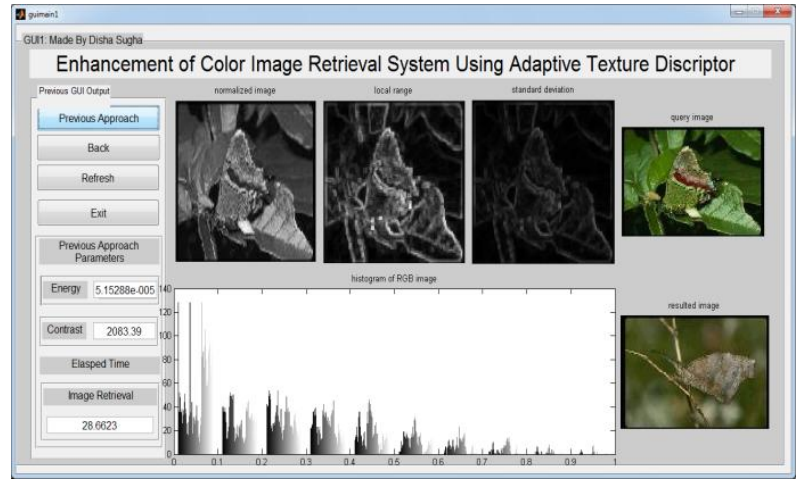

Fig3. GUI2 previous approach

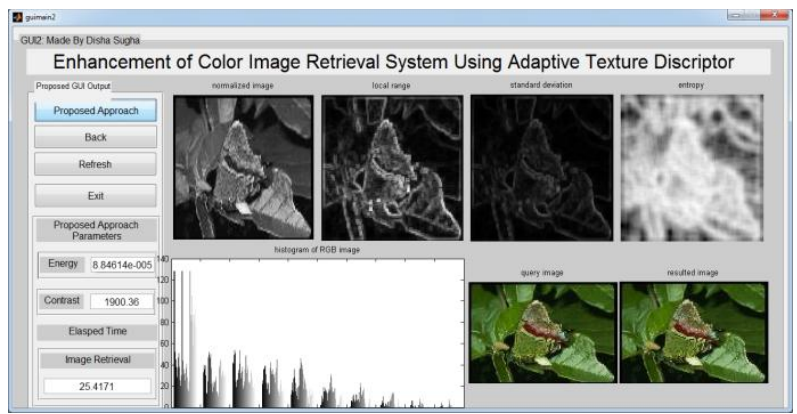

Fig4. GUI 2 proposed approach

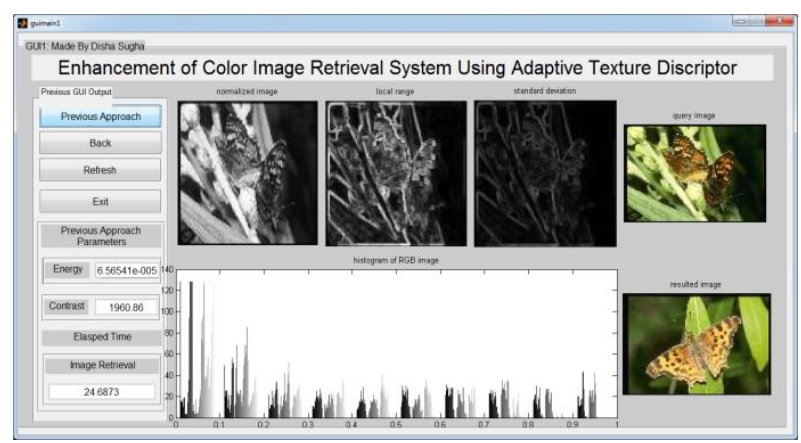

Fig5. GUI3 previous approach

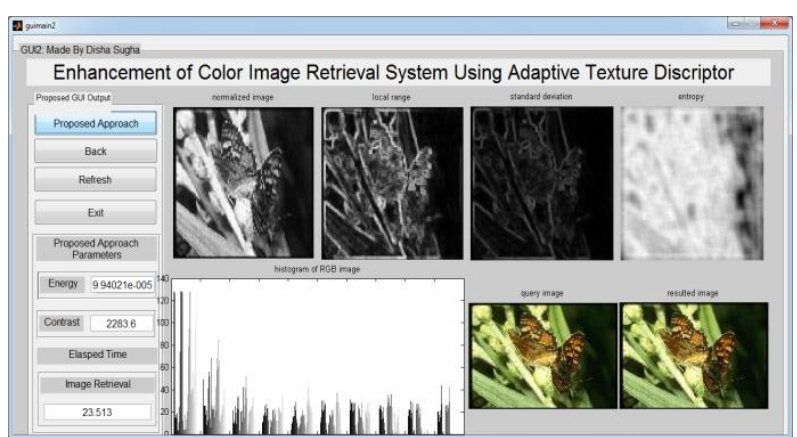

Fig6. GUI3 proposed approach

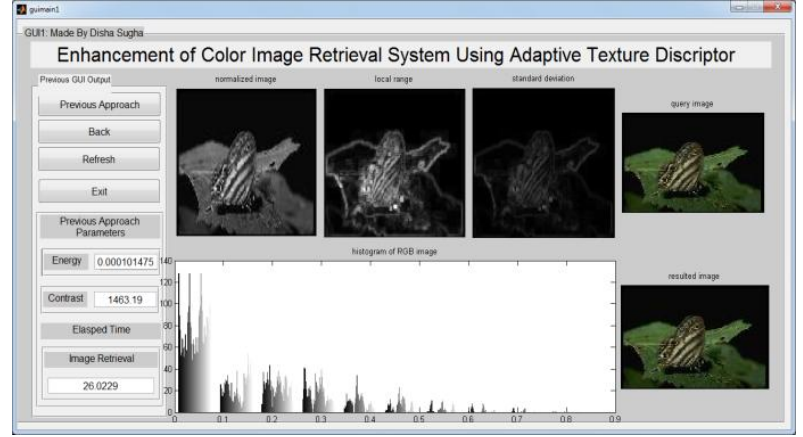

Fig7. GUI4 previous approach

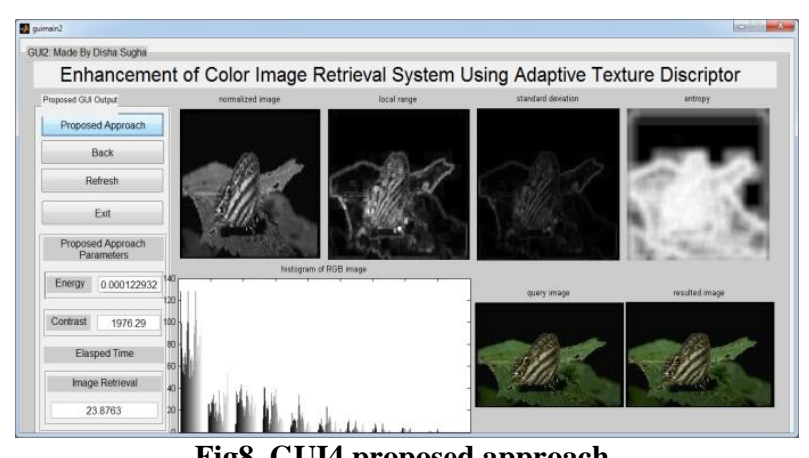

Fig8. GUI4 proposed approach

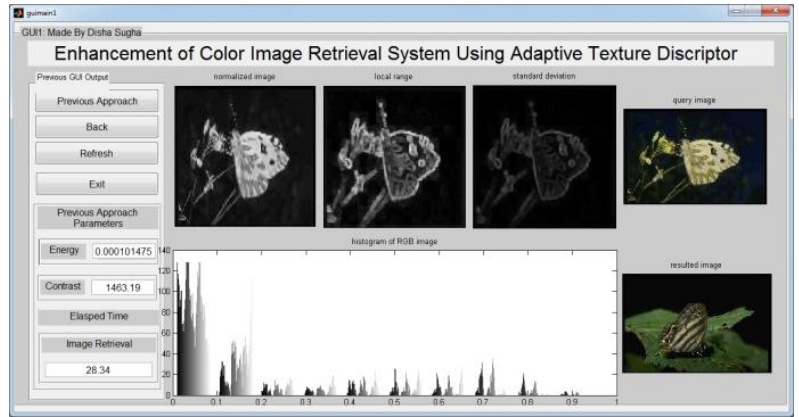

Fig9. GUI5 previous approach

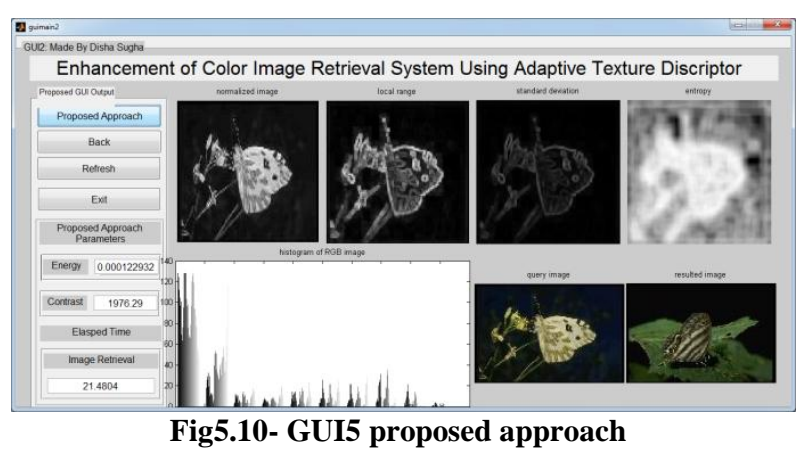

\section{CONCLUSION}

On the basis of previous researches, this paper consists of calculation on three parameters, which are energy, contrast and time taken to retrieve the image. On the basis of these textures retrieved images from the database and give the retrieved time of images. It gives the best matchingimage from the database. For the future work using different type of features to enhance the pixel value of image and give the better image in result and can also improve the retrieved time of images [5]. 


\section{REFERENCES}

[1] V. Ramya, 2018 "Content based image retrieval system using clustering with combined patterns", International Journal of Scientific Research in Computer Science Engineering and Information Technology, vol3, issue1, ISSN:2456-3307.

[2] Aasia Ali and Sanjay Sharma, 2017 "Content Based Image Retrieval using feature Extraction with Machine Learning", International Conference on Intelligent Computing and Control Systems ICICCS.

[3] Vishal Lonarkar and Ashwath Rao B, 2017 "ContentBased Image Retrieval by segmentation and Clustering", International Conference on Inventive Computing and Informatics (ICICI 2017) IEEE -Part number: CFP17L34-ART, ISBN:978-1-5386-4031-9.

[4] S.Banuchitra, K.Kungumaraj, 2016 "A Comprehensive survey of content based image retrieval techniques", International journal of engineering and computer science ISSN:2319-7242, volume 5 issues 8 Aug 2016, page no. 17577-17584.
[5] Apurva N.Ganar, C.S.Gode, Sachin M. Jambhulkar, 2014 "Enhancement of image retrieval by using color, texture and shape features", international conference on Electronic systems, signal processing and computing technologies.

[6] V.anusha, Usha Reddy, T.Ramashri, 2014, "Content based image retrieval using colour moments and texture", International journal of engineering research \& technology,ISSN:2278-0181,Vol.3 Issue 2.

[7] Sonali Bhadoria, Meenakshi Madugunki, C.G.Dethe, Preeti Aggarwal, 2011, "Comparison of Color, texture and ICM features in CBIR system, International conference on control, Robotics and Cybernetics (ICCRC 2011).

[8] Haiyu Song, Xiongfei Li and Pengie Wang, 2010 "Adaptive Feature Selection and Extraction Approaches for Image. 